\title{
Direct Downconversion of Multiple RF Signals Using Bandpass Sampling
}

\author{
Ching-Hsiang Tseng and Sun-Chung Chou \\ Department of Electrical Engineering \\ National Taiwan Ocean University \\ 2 Pei-Ning Rd., Keelung, Taiwan, R.O.C.
}

\begin{abstract}
The center idea behind the software radio architecture is to place analog-to-digital and digital-to-analog converters as near the antenna as possible, leaving the implementation of the most radio functionality to a programmable micro or signal processor. One way to accomplish this in a radio receiver front end is by direct downconverting the desired radio frequency (RF) signal to a target intermediate frequency (IF) using bandpass sampling. Although the bandpass sampling theory for a single RF signal is well developed, its counterpart for two or more RF signals is relatively immature. For direct downconverting multiple distinct RF signals, determining valid bandpass sampling frequencies using the conventional approach could be a computationally exhaustive process. In this paper, we propose an efficient method to find the ranges of valid bandpass sampling frequency for direct downconverting multiple distinct RF signals. Simple formulas for the ranges of valid bandpass sampling frequency in terms of bandwidths and frequency locations of the RF signals are derived. The result can be used to efficiently choose an appropriate bandpass sampling frequency for multiple RF signals.
\end{abstract}

\section{INTRODUCTION}

The main design philosophy of the software radio is to allow the most radio functionality be realized in the programmable digital domain via digital signal processors (DSPs) [1], whereby achieves the highest design flexibility. Bearing this in mind, one should, in designing a receiver front end, push the analog-to-digital converter (ADC) as close to the antenna as possible. One way to do this is by wideband digitizing the radio signal at the RF using an ADC [2]. This design eliminates the requirement of using analog mixers for frequency downconversion. However, for most radio applications, the required sampling rate for the ADC would be impractically high if Nyquist sampling [3] is employed. For example, the GSM (Global System for Mobile Communication) 1800 digital cellular telecommunication system uses carrier frequencies of about $1.8 \mathrm{GHz}$ and has a bandwidth of $200 \mathrm{kHz}$ per channel [4]. Therefore, Nyquist sampling a GSM 1800 signal requires a sampling frequency of about $3.6 \mathrm{GHz}$. Such a high sampling rate could still be infeasible with present technology. Alternatively, by aware of the bandpass nature of the radio signal, one can use bandpass sampling instead to directly downconvert the desired RF signal to an IF. According to the bandpass sampling theory [5], the minimum sampling rate is dependent on the bandwidth, but not the maximum frequency, of the RF signal. Using the GSM 1800 signal as an example, the minimum sampling rate is only about $400 \mathrm{kHz}$ if bandpass sampling is employed. This significantly relaxes the demand for fast ADCs and DSPs. The lower processing rate could in turn reduce the power consumption, which is of importance for mobile devices.

To fully exploit the benefits of the software radio, direct downconversion of multiple RF signals using a single receiver front end may be desirable. In this regard, a method to determine the bandpass sampling frequency for direct digitization of multiple RF signals has been proposed in [6], where constraints on the sampling frequency for avoiding aliasing were developed. This method, however, is computationally intensive because exhaustive tests on the constraints for all frequencies up to the Nyquist rate would be required. The computational complexity can be alleviated to certain extent by using the method presented in [7], where a modified interpretation to a graph showing allowable sampling frequencies for all desired RF signals was proposed. This method finds the valid sampling frequency ranges for each RF signal and shows the result with a graph. By overlapping the graphs of valid sampling frequency ranges for all RF signals, one can obtain the intersection of the graphs. Only those frequencies within the frequency ranges of the intersection need to be tested for their validity, thereby reduces the required computational complexity.

In this paper, a novel method is proposed to obtain valid sampling frequency ranges for direct downconversion of multiple RF signals. In the proposed method, we first determine all the possible orders of spectral replicas in the spectrum of the sampled signal. For each possible order of spectral replicas, we derive the formula for its valid sampling frequency range in terms of some parameters (such as bandwidths and locations of the RF signals, etc). For a given problem, one only needs to substitute the relevant parameters into the formulas to obtain the valid sampling frequency ranges for direct downconversion of the desired RF signals. Compared to the conventional approach, the proposed method is superior in sense of both computational complexity and ease of implementation. Computer simulation on sampling GSM signals is conducted to demonstrate the usage of the proposed method.

\section{BANDPASS SAMPLing of A Single RF Signal}

Consider sampling an RF signal whose spectrum is shown in Fig. 1(a). Let the sampling frequency be $f_{s} \mathrm{~Hz}$, then the spec- 


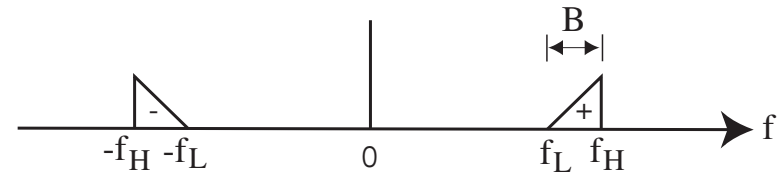

(a)

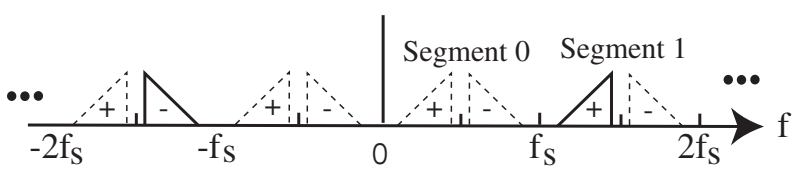

(b)

Fig. 1. The spectra of (a) the original and (b) the sampled bandpass signals.

trum of the sampled signal can be obtained by replicating the spectrum of the original signal at multiples of $f_{s}$. According to the bandpass sampling theory, the spectrum of the original signal must not straddle $m \frac{f_{s}}{2}$ for any integer $m$, otherwise the sampled signal would be aliased [8]. Therefore, for aliasingfree sampling, the entire positive-frequency spectrum of the original signal (labeled ' + ' in Fig. 1(a)) must lie in the frequency range $\left[m \frac{f_{s}}{2},(m+1) \frac{f_{s}}{2}\right]$ for some integer $m$. An example for the case of the positive-frequency spectrum lying in the frequency range $\left[\frac{2 f_{s}}{2}, \frac{3 f_{s}}{2}\right]$ (i.e., $m=2$ ) is shown in Fig. 1(b), where solid and dashed lines are used for the original spectra and the replicas, respectively. From Fig. 1(b) we see that, in the frequency segment $\left[n f_{s},(n+1) f_{s}\right]$ for any integer $n$ (referred to as segment $n$ ), the center frequencies of the positive-frequency and negative-frequency replicas (labeled '+' and '-' respectively in Fig. 1(b)) are symmetric with respect to $\left(n+\frac{1}{2}\right) f_{s}$. This property will be used in the following section.

By using bandpass sampling, an RF signal centered at $f_{c}$ will be downconverted to an intermediate frequency $f_{\mathrm{IF}}$. The $f_{\mathrm{IF}}$ is related to $f_{c}$ and $f_{s}$ as follows [6]:

$$
f_{\mathrm{IF}}= \begin{cases}\operatorname{rem}\left(f_{c}, f_{s}\right), & \text { if }\left\lfloor\frac{f_{c}}{f_{s} / 2}\right\rfloor \text { is even } \\ f_{s}-\operatorname{rem}\left(f_{c}, f_{s}\right), & \text { if }\left\lfloor\frac{f_{c}}{f_{s} / 2}\right\rfloor \text { is odd }\end{cases}
$$

where $\operatorname{rem}(a, b)$ denotes the remainder of $a$ divided by $b$, and $\lfloor x\rfloor$ denotes the largest integer less than or equal to $x$. Note that, depending on the value of $\left\lfloor\frac{f_{c}}{f_{s} / 2}\right\rfloor$ (even or odd), either the positive or the negative spectrum of the original signal will be downconverted to $f_{\mathrm{IF}}$.

\section{BAndpass SAMPling OF Two RF Signals}

Consider the problem of sampling the two RF signals shown in Fig. 2. The signal ' $i$ ' ( $i=1$ or 2$)$ is labeled ' $i$ '. Its lowest, center, and highest frequencies are denoted by $f_{L_{i}}, f_{i}$, and $f_{H_{i}}$, respectively. Its bandwidth is given by $B_{i}=f_{H_{i}}-f_{L_{i}}$. The center frequencies of the two RF signals are related by $f_{2}=R f_{1}$, where $R$ is a positive real number.

Let the sampling frequency be $f_{s}$. According to Section II, the spectrum of each signal must not straddle any integer multiple of $f_{s} / 2$, otherwise the sampled signal would be aliased. Therefore, the positive spectra of signals ' 1 ' and ' 2 '

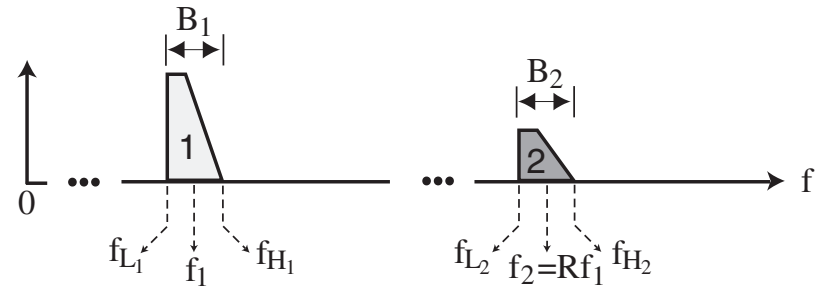

Fig. 2. The spectrum of two RF signals.

must be inside some segments $n_{1}$ and $n_{2}$, respectively. In addition, the positive spectrum of each signal may not straddle the midpoint of its corresponding segment, it can only be completely inside either the first or the second half of the segment.

Since the sampling frequency is $f_{s} \mathrm{~Hz}$, the spectrum of the sampled signal can be obtained by replicating the spectrum of the original signal at multiples of $f_{s}$. Due to spectral replication, a replica of spectrum ' $i$ ' $(i= \pm 1, \pm 2)$ would appear in each segment. To be immune from aliasing, the replicas in each segment can not overlap. From Section II and Fig. 1(b) we know that the relative positions of the replicas ' 1 ' and ' -1 ' in each segment will be symmetric with respect to the midpoint of the segment, so will the relative positions of the replicas '2' and '-2' in each segment. That is, each RF signal must have its positive or negative replica inside the first half of a segment. Since the two replicas inside the first half segment must not overlap, there are two choices for ordering the two replicas. The total number of possible replica orders in one segment is thus $2 \times 2 \times 2=8$. The spectra of the sampled signal for the 8 possible replica orders are shown in Fig. 3, where we use solid trapezoids to denote the spectra of the original signals and dashed trapezoids to denote the replicas. The label on each trapezoid indicates the source of the spectrum. For example, a dashed trapezoid labeled '-1' denotes a replica of the negative spectrum of signal ' 1 '.

Note that, as shown in Figs. 3(a) and (b), the replicas ' 1 ' and '2' are neighbors in the first half of a segment in Case 1 and in the second half of a segment in Case 2. If those cases having the same neighboring replicas in either the first or the second half of a segment are considered as in one group, we may categorize the 8 cases in Fig. 3 into 4 groups, as shown in Table I. Each group contains two cases, and the two cases share the same neighboring replicas in either the first or the second half of a segment.

In the following, we will derive the ranges of valid bandpass sampling frequency for the 8 cases shown in Fig. 3. For a given replica order, the sampling frequency must satisfy certain constraints. There are two types of constraints: one is referred to as neighbor constraint and the other is referred to as boundary constraint. The two cases in each group have the same neighboring replicas and thus share the same neighbor constraint. However, they have different boundary constraints because the neighboring replicas show up in different halves of a segment. 

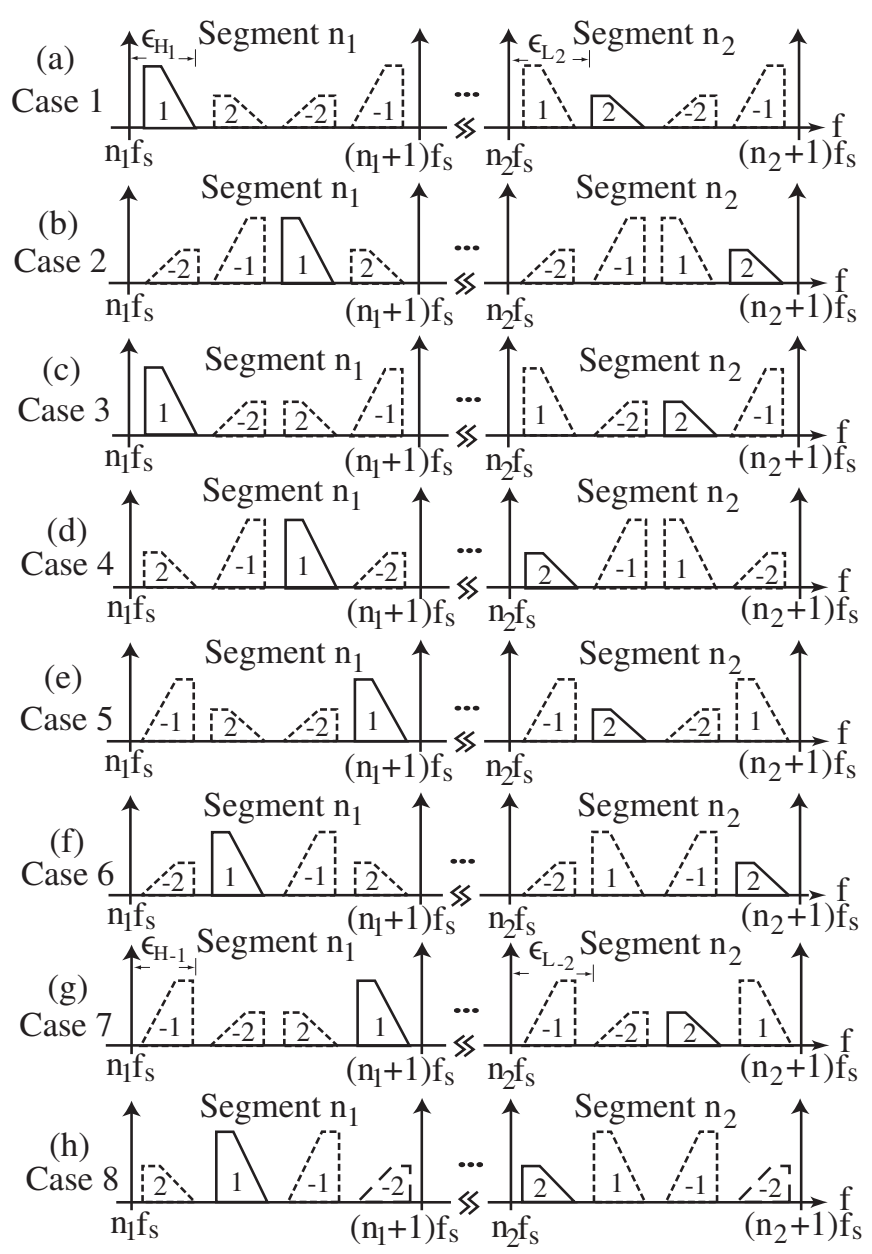

Fig. 3. The spectrum of the two RF signals shown in Fig. 2 after bandpass sampling. The 8 possible replica orders are shown in (a) to (h). The parameters $\epsilon_{L_{i}}(i= \pm 2)$ and $\epsilon_{H_{i}}(i= \pm 1)$ are defined in (a) and $(\mathrm{g})$.

Let's consider the neighbor constraint first. From Table I we see that the neighboring replicas in Group 1 are $(1,2)$. Therefore, in each segment, the relative highest frequency of replica ' 1 ' must be less than or equal to the relative lowest frequency of replica '2'. For simplicity, we define $\epsilon_{L_{i}}(i= \pm 2)$ and $\epsilon_{H_{i}}(i= \pm 1)$ as the relative lowest and highest frequencies of replica ' $i$ ' in a segment, as shown in Figs. 3(a) and 3(g). Based on the definitions in Figs. 3(a) and 3(g), one can easily obtain the values of $\epsilon_{L_{i}}$ and $\epsilon_{H_{i}}$. The result is shown in Table II. Using these definitions, we may write the neighbor constraint for Group 1 with a shorthand representation as $\epsilon_{H_{1}}<\epsilon_{L_{2}}$, which can lead to

$$
f_{s} \leq \frac{f_{L_{2}}-f_{H_{1}}}{n_{2}-n_{1}}
$$

with the aid of Table II. Following the same procedure, one can easily find the shorthand representations of the neighbor constraints and their corresponding inequalities for the other three groups. The result is summarized in Table I.

The boundary constraint for each case can be obtained by observing Figs. 2 and 3. Taking Case 1 as an example, the
TABLE I

THE CATEgorization OF THE 8 CASES IN Fig. 3 Into 4 GROUPS. THE SHORTHAND REPRESENTATIONS AND THEIR CORRESPONDING NEIGHBOR CONSTRAINTS FOR THE 4 GROUPS ARE ALSO SHOWN.

\begin{tabular}{c|c|c|c|c} 
Group & Neighbors & Case & Shorthand & Constraint \\
\hline 1 & $(1,2)$ & 1 & $\epsilon_{H_{1}} \leq \epsilon_{L_{2}}$ & $f_{s} \leq \frac{f_{L_{2}}-f_{H_{1}}}{n_{2}-n_{1}}$ \\
\hline 2 & $(1,-2)$ & 3 & $\epsilon_{H_{1}} \leq \epsilon_{L_{-2}}$ & $f_{s} \geq \frac{f_{H_{1}}+f_{H_{2}}}{n_{1}+n_{2}+1}$ \\
\hline 3 & $(-1,2)$ & 5 & $\epsilon_{H_{-1}} \leq \epsilon_{L_{2}}$ & $f_{s} \leq \frac{f_{L_{1}}+f_{L_{2}}}{n_{1}+n_{2}+1}$ \\
\hline 4 & $(-1,-2)$ & 7 & $\epsilon_{H_{-1}} \leq \epsilon_{L_{-2}}$ & $f_{s} \geq \frac{f_{H_{2}}-f_{L_{1}}}{n_{2}-n_{1}}$
\end{tabular}

TABLE II

THE FREQUENCY VALUES OF $\epsilon_{L_{i}}(i= \pm 2)$ AND $\epsilon_{H_{i}}(i= \pm 1)$.

\begin{tabular}{c|c} 
Notation & Frequency Value \\
\hline$\epsilon_{H_{1}}$ & $f_{H_{1}}-n_{1} f_{s}$ \\
$\epsilon_{H_{-1}}$ & $\left(n_{1}+1\right) f_{s}-f_{L_{1}}$ \\
$\epsilon_{L_{2}}$ & $f_{L_{2}}-n_{2} f_{s}$ \\
$\epsilon_{L_{-2}}$ & $\left(n_{2}+1\right) f_{s}-f_{H_{2}}$
\end{tabular}

neighboring replicas $(1,2)$ must be completely inside the first half of a segment. We therefore see from Figs. 2 and 3(a) that the boundary constraints for spectra ' 1 ' and ' 2 ' are

$$
\begin{aligned}
& f_{L_{1}} \geq n_{1} f_{s} \\
& f_{H_{2}} \leq\left(n_{2}+\frac{1}{2}\right) f_{s},
\end{aligned}
$$

respectively. For Case 2, the boundary constraint only differs from Case 1 in that the neighboring replicas $(1,2)$ are now in the second half instead of the first half of a segment. Therefore, the boundary constraints for spectra ' 1 ' and ' 2 ' can be obtained from (3) and (4) by replacing $n_{1}$ and $n_{2}$ with $n_{1}+\frac{1}{2}$ and $n_{2}+\frac{1}{2}$, respectively. This leads to

$$
\begin{aligned}
& f_{L_{1}} \geq\left(n_{1}+\frac{1}{2}\right) f_{s} \\
& f_{H_{2}} \leq\left(n_{2}+1\right) f_{s} .
\end{aligned}
$$

Following the same procedure, it is straightforward to derive the boundary constraints for all other cases. The result is listed in Table III.

A valid sampling frequency must satisfy both the neighbor and boundary constraints. By combining Tables I and III, one obtains Table IV, where the ranges of valid sampling frequency for the 8 cases in Fig. 3 are shown.

\section{Finding An Appropriate Range of Valid SAMPLING FREQUENCY}

From Table IV we see that all the parameters required to determine the ranges of valid sampling frequency are given in Fig. 2 except the integers $n_{1}$ and $n_{2}$. Since $n_{1}$ is the number of the segment where spectrum ' 1 ' is located, we know that $n_{1}=\left\lfloor\frac{f_{L_{1}}}{f_{s}}\right\rfloor$. This indicates that the larger the value of $n_{1}$, the 
TABLE III

THE BOUNDARY CONSTRAINTS FOR THE 8 CASES IN FIG. 3.

\begin{tabular}{c|c|c}
\multirow{2}{*}{ Case } & \multicolumn{2}{|c}{ Boundary Constraint } \\
\cline { 2 - 3 } & Spectrum '1' & Spectrum '2' \\
\hline 1 & $f_{L_{1}} \geq n_{1} f_{s}$ & $f_{H_{2}} \leq\left(n_{2}+\frac{1}{2}\right) f_{s}$ \\
2 & $f_{L_{1}} \geq\left(n_{1}+\frac{1}{2}\right) f_{s}$ & $f_{H_{2}} \leq\left(n_{2}+1\right) f_{s}$ \\
3 & $f_{L_{1}} \geq n_{1} f_{s}$ & $f_{L_{2}} \geq\left(n_{2}+\frac{1}{2}\right) f_{s}$ \\
4 & $f_{L_{1}} \geq\left(n_{1}+\frac{1}{2}\right) f_{s}$ & $f_{L_{2}} \geq n_{2} f_{s}$ \\
5 & $f_{H_{1}} \leq\left(n_{1}+1\right) f_{s}$ & $f_{H_{2}} \leq\left(n_{2}+\frac{1}{2}\right) f_{s}$ \\
6 & $f_{H_{1}} \leq\left(n_{1}+\frac{1}{2}\right) f_{s}$ & $f_{H_{2}} \leq\left(n_{2}+1\right) f_{s}$ \\
7 & $f_{H_{1}} \leq\left(n_{1}+1\right) f_{s}$ & $f_{L_{2}} \geq\left(n_{2}+\frac{1}{2}\right) f_{s}$ \\
8 & $f_{H_{1}} \leq\left(n_{1}+\frac{1}{2}\right) f_{s}$ & $f_{L_{2}} \geq n_{2} f_{s}$
\end{tabular}

TABLE IV

THE RANGES OF VALID SAMPLING FREQUENCY FOR THE 8 CASES.

\begin{tabular}{c|c} 
Case & Range of Valid $f_{s}$ \\
\hline 1 & $\frac{f_{H_{2}}}{n_{2}+\frac{1}{2}} \leq f_{s} \leq \min \left\{\frac{f_{L_{1}}}{n_{1}}, \frac{f_{L_{2}}-f_{H_{1}}}{n_{2}-n_{1}}\right\}$ \\
2 & $\frac{f_{H_{2}}}{n_{2}+1} \leq f_{s} \leq \min \left\{\frac{f_{L_{1}}}{n_{1}+\frac{1}{2}}, \frac{f_{L_{2}}-f_{H_{1}}}{n_{2}-n_{1}}\right\}$ \\
3 & $\frac{f_{H_{1}}+f_{H_{2}}}{n_{1}+n_{2}+1} \leq f_{s} \leq \min \left\{\frac{f_{L_{1}}}{n_{1}}, \frac{f_{L_{2}}}{n_{2}+\frac{1}{2}}\right\}$ \\
4 & $\frac{f_{H_{1}}+f_{H_{2}}}{n_{1}+n_{2}+1} \leq f_{s} \leq \min \left\{\frac{f_{L_{1}}}{n_{1}+\frac{1}{2}}, \frac{f_{L_{2}}}{n_{2}}\right\}$ \\
5 & $\max \left\{\frac{f_{H_{1}}}{n_{1}+1}, \frac{f_{H_{2}}}{n_{2}+\frac{1}{2}}\right\} \leq f_{s} \leq \frac{f_{L_{1}}+f_{L_{2}}}{n_{1}+n_{2}+1}$ \\
6 & $\max \left\{\frac{f_{H_{1}}}{n_{1}+\frac{1}{2}}, \frac{f_{H_{2}}}{n_{2}+1}\right\} \leq f_{s} \leq \frac{f_{L_{1}}+f_{L_{2}}}{n_{1}+n_{2}+1}$ \\
7 & $\max \left\{\frac{f_{H_{1}}}{n_{1}+1}, \frac{f_{H_{2}}-f_{L_{1}}}{n_{2}-n_{1}}\right\} \leq f_{s} \leq \frac{f_{L_{2}}}{n_{2}+\frac{1}{2}}$ \\
8 & $\max \left\{\frac{f_{H_{1}}}{n_{1}+\frac{1}{2}}, \frac{f_{H_{2}}-f_{L_{1}}}{n_{2}-n_{1}}\right\} \leq f_{s} \leq \frac{f_{L_{2}}}{n_{2}}$
\end{tabular}

smaller the value of the sampling frequency $f_{s}$. However, the bandwidth of a segment should at least accommodate the 4 nonoverlapping replicas (i.e., replicas '1', '-1', '2', and '-2'). This means $f_{s} \geq 2\left(B_{1}+B_{2}\right)$. Therefore, the value of $n_{1}$ is upper bounded by

$$
n_{1}=\left\lfloor\frac{f_{L_{1}}}{f_{s}}\right\rfloor \leq\left\lfloor\frac{f_{L_{1}}}{2\left(B_{1}+B_{2}\right)}\right\rfloor
$$

In most situations, one would prefer choosing a smaller sampling frequency to lower the processing rate. Therefore, the largest integer $n_{1}$ satisfying (7) should be a good starting point for searching the ranges of valid sampling frequency. In addition, since spectrum ' 1 ' is in segment $n_{1}$, one can write

$$
n_{1} f_{s}<f_{1}<\left(n_{1}+1\right) f_{s} .
$$

Multiplying (8) by $\frac{R}{f_{s}}$ and using the relation of $f_{2}=R f_{1}$, one obtains

$$
R n_{1}<\frac{f_{2}}{f_{s}}<R\left(n_{1}+1\right)
$$

Taking \lfloor\rfloor on (9) leads to

$$
\left\lfloor R n_{1}\right\rfloor \leq n_{2} \leq\left\lfloor R n_{1}+R\right\rfloor,
$$

where the fact that spectrum ' 2 ' is in segment $n_{2}$ and thus $n_{2}=\left\lfloor\frac{f_{2}}{f_{s}}\right\rfloor$ has been used in deriving (10). This suggests that, for a given $n_{1}$, the possible values of $n_{2}$ are confined by (10).
In typical situations, the value of $R$ is not large, which makes the range of $n_{2}$ relatively small.

Based on the above analysis, we may summarize the procedure for obtaining a range of valid sampling frequency for two RF signals as follows:

1) Choose an appropriate $n_{1}$ using (7).

2) Choose an appropriate $n_{2}$ using (10).

3) Use Table IV to find the ranges of valid sampling frequency.

As mentioned earlier, the larger the value of $n_{1}$, the smaller the value of the sampling frequency. Therefore, one might want to choose a relatively large value of $n_{1}$ which satisfies (7) to obtain a low sampling frequency. Note that (7) and (10) are only theoretical bounds on $n_{1}$ and $n_{2}$, respectively. In practice a choice of $\left(n_{1}, n_{2}\right)$ satisfying them does not necessarily guarantee a solution. In addition, for a particular choice of $\left(n_{1}, n_{2}\right)$, some of the 8 cases may yield sampling frequency ranges which are empty. When that happens, it simply means that there is no valid sampling frequency available for the particular replica order under the chosen $\left(n_{1}, n_{2}\right)$.

\section{COMPuter Simulation - BANDPASS SAMPling OF Two GSM SIGNALS}

To demonstrate the usage of the proposed method, we consider a mobile station receiving signals from GSM 900 and GSM 1800 base stations. For the base to mobile link, the GSM 900 system operates in the band of $935-960 \mathrm{MHz}$, while the GSM 1800 system operates in the bnad of 1805 $1880 \mathrm{MHz}$. The bandwidth per channel for both systems is $200 \mathrm{KHz}$. In this simulation, it is assumed that receiving the GSM 900 signal from the channel $935.2-935.4 \mathrm{MHz}$ and the GSM 1800 signal from the channel $1805.2-1805.4 \mathrm{MHz}$ is desirable for the mobile station. The GSM 900 signal was simulated by passing a wideband noise through a 4-th order Butterworth bandpass filter with a passband of 935.2-935.4 $\mathrm{MHz}$, while the GSM 1800 signal was simulated by passing the same wideband noise through a 4-th order Butterworth bandpass filter with a passband of 1805.2-1805.4 MHz. The two GSM signals were then added up to obtain the simulated received signal for the mobile station. The power spectrum of this signal is plotted in Fig. 4, where two bands centering at 935.3 MHz and 1805.3 MHz are clearly shown.

For the given problem, we have $f_{L_{1}}=935.2 \mathrm{MHz}, f_{H_{1}}=$ $935.4 \mathrm{MHz}, f_{L_{2}}=1805.2 \mathrm{MHz}, f_{H_{2}}=1805.4 \mathrm{MHz}, B_{1}=$ $B_{2}=200 \mathrm{kHz}$, and $R=1805.3 / 935.3=1.93$. According to (7), the value of $n_{1}$ is upper bounded by

$$
n_{1} \leq\left\lfloor\frac{935.2}{2(0.2+0.2)}\right\rfloor=1169 .
$$

For those $n_{1}$ which satisfies (11), we used (10) to determine the possible values of $n_{2}$. For each possible combination of $\left(n_{1}, n_{2}\right)$, we substituted the relevant parameters into the formulas in Table IV to obtain the ranges of valid bandpass sampling frequency for the 8 cases. In Table $\mathrm{V}$, we show the 10 lowest frequency ranges of valid sampling frequency we found using the proposed method. Note that, although the theoretical 


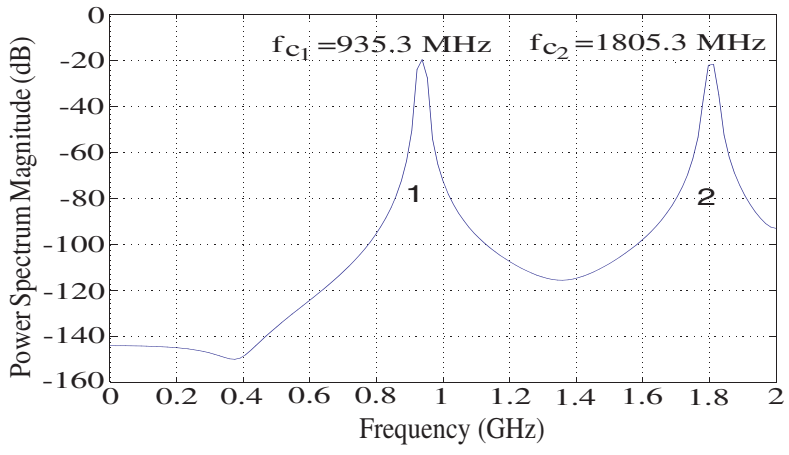

Fig. 4. The original spectrum of the simulated GSM signal.

TABLE V

THE TEN LOWEST FREQUENCY RANGES OF VALID $f_{s}$.

\begin{tabular}{c|c|c|c} 
case & $n_{1}$ & $n_{2}$ & Range of Valid $f_{s}(\mathrm{~Hz})$ \\
\hline 2 & 1122 & 2166 & $833133.4 \leq f_{s} \leq 833140.3$ \\
5 & 1122 & 2167 & $832947.5 \leq f_{s} \leq 832948.3$ \\
3 & 1126 & 2173 & $830545.5 \leq f_{s} \leq 830549.8$ \\
6 & 1129 & 2179 & $828165.2 \leq f_{s} \leq 828165.6$ \\
4 & 1133 & 2188 & $825045.2 \leq f_{s} \leq 825045.7$ \\
5 & 1136 & 2194 & $822693.1 \leq f_{s} \leq 822695.8$ \\
8 & 1140 & 2201 & $820169.7 \leq f_{s} \leq 820172.6$ \\
1 & 1144 & 2208 & $817478.0 \leq f_{s} \leq 817481.2$ \\
4 & 1147 & 2215 & $814986.7 \leq f_{s} \leq 814988.7$ \\
6 & 1158 & 2235 & $807424.0 \leq f_{s} \leq 807424.8$
\end{tabular}

upper bound for $n_{1}$ is 1169 , no solution can be found until $n_{1}$ goes down to 1158 , where the Case 6 for $n_{2}=2235$ yields a valid sampling frequency range of [807424.0,807424.8] Hz. Sampling frequencies in this range are slightly larger than the theoretical minimum sampling frequency of $2(200+200)=800$ $\mathrm{kHz}$. Also notice that, for $\left(n_{1}, n_{2}\right)=(1158,2235)$, only Case 6 appears in Table V. This is because that for such a large value of $n_{1}$ the bandwidth of a segment is too small to accommodate the 4 nonoverlapping replicas with other particular orders. This phenomenon happens to other choices of $\left(n_{1}, n_{2}\right)$ listed in Table $\mathrm{V}$ as well. The smaller the $n_{1}$ value, the better chance a particular case can yield a solution.

One may have noticed that the ranges of valid $f_{s}$ listed in Table $\mathrm{V}$ are very narrow. If one choose the sampling frequency based on Table V, a slight frequency offset of the sampling circuit could drive the sampling frequency out of the valid range. In actual implementation, one might want to choose a valid sampling frequency which corresponds to a smaller $n_{1}$. For demonstration purpose, we chose Case 8 for $\left(n_{1}, n_{2}\right)=$ $(352,680)$ as an example. In this case, we obtained the valid $f_{s}$ range of $[2653617,2654705] \mathrm{Hz}$. We selected $f_{s}=2654280$ $\mathrm{Hz}$ to sample the simulated GSM signal. With this choice of $f_{s}$, one can use (1) to calculate the intermediate frequencies, say $f_{\mathrm{IF}_{1}}$ and $f_{\mathrm{IF}_{2}}$, for the two GSM signals as follows:

$$
\begin{aligned}
f_{\mathrm{IF}_{1}}= & \operatorname{rem}(935300000,2654280)=993440 \mathrm{~Hz}, \\
& \left\lfloor\frac{935300000}{2654280 / 2}\right\rfloor=704(\text { even }) \\
f_{\mathrm{IF}_{2}=}= & \operatorname{rem}(1805300000,2654280)=389600 \mathrm{~Hz},
\end{aligned}
$$

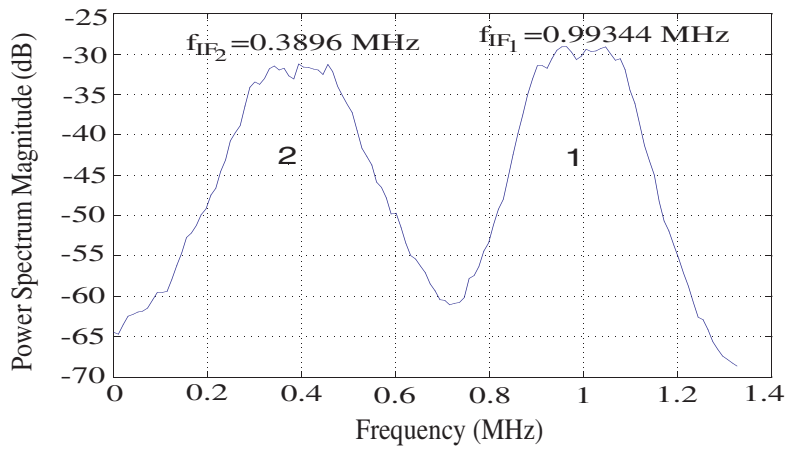

Fig. 5. The spectrum of the sampled GSM signal.

$$
\left\lfloor\frac{1805300000}{2654280 / 2}\right\rfloor=1360 \text { (even) }
$$

Note that positive spectra of the two GSM signals are downconverted to $f_{\mathrm{IF}_{1}}$ and $f_{\mathrm{IF}_{2}}$, because $\left\lfloor\frac{f_{c}}{f_{s} / 2}\right\rfloor$ is even in both (12) and (13). The power spectrum of the sampled signal is shown in Fig. 5, where two nonoverlapping bands centering at $f_{\mathrm{IF}_{2}}=0.3896 \mathrm{MHz}$ and $f_{\mathrm{IF}_{1}}=0.99344 \mathrm{MHz}$ are clearly shown. This result matches the replica order given by Case 8 in Fig. 3(h).

\section{CONCLUSION}

In this paper, we have presented an efficient method to determine the ranges of valid bandpass sampling frequency for two distinct RF signals. We have derived formulas for the ranges of valid bandpass sampling frequency in terms of bandwidths and frequency locations of the RF signals. Although the developed formulas are for two RF signals only, extension of the derivation to three or higher number of RF signals is straightforward. The simplicity of this method guarantees its easy implementation on a microprocessor.

\section{ACKNOWLEDGMENT}

This work was supported in part by the National Science Council under Contract NSC 91-2213-E-019-009.

\section{REFERENCES}

[1] Joe Mitola, "The software radio architecture," IEEE Commun. Mag., vol. 33, no. 5, pp. 26-38, May 1995.

[2] Jeffery A. Wepman, "Analog-to-digital converters and their applications in radio receivers," IEEE Commun. Mag., vol. 33, no. 5, pp. 39-45, May 1995.

[3] Simon Haykin, Communication Systems. 4th ed., New York : John Wiley \& Sons, 2001.

[4] Digital Cellular Telecommunications System (Phase 2+); Radio Transmission and Reception (GSM 05.05 Version 8.5.1 Release 1999), ETSI EN 300910 Ver. 8.5.1 (2000-11).

[5] R. G. Vaughan, N. L. Scott, and D. R. White, "The theory of bandpass sampling," IEEE Trans. on Signal Processing, vol. 39, no. 9, pp.19731984, Sep 1991.

[6] D. M. Akos, M. Stockmaster, J. B. Y. Tsui, and J. Caschera, "Direct bandpass sampling of multiple distinct RF signals," IEEE Trans. Commun., vol. 47, no. 7, pp. 983-988, July 1999.

[7] N. Wong and T.-S. Ng, "An efficient algorithm for downconverting multiple bandpass signals using bandpass sampling," Proc. IEEE Int. Conf. Commun., Helsinki, Finland, June 2001, vol. 3, pp.910-914.

[8] C.-H. Tseng, "Bandpass sampling criteria for nonlinear systems," IEEE Trans. Signal Processing, vol. 50, no. 3, pp. 568-577, March 2002. 